MATHEMATICS OF COMPUTATION

Volume 76, Number 257, January 2007, Pages 299-321

S $0025-5718(06) 01894-1$

Article electronically published on August 8, 2006

\title{
SHEPARD-BERNOULLI OPERATORS
}

\author{
R. CAIRA AND F. DELL'ACCIO
}

\begin{abstract}
We introduce the Shepard-Bernoulli operator as a combination of the Shepard operator with a new univariate interpolation operator: the generalized Taylor polynomial. Some properties and the rate of convergence of the new combined operator are studied and compared with those given for classical combined Shepard operators. An application to the interpolation of discrete solutions of initial value problems is given.
\end{abstract}

\section{THE PROBLEM}

Let $X=\left\{x_{1}, \ldots, x_{N}\right\}$ be a set of $N$ distinct points of $\mathbb{R}^{s}, s \in \mathbb{N}$, and let $f$ be a function defined on a domain $D$ containing $X$. The classical Shepard operators (first introduced in [24] in the particular case $s=2$ ) are defined by

$$
S_{N, \mu}[f](x)=\sum_{i=1}^{N} A_{\mu, i}(x) f\left(x_{i}\right), \quad \mu>0,
$$

where the weight functions $A_{\mu, i}(x)$ in barycentric form are

$$
A_{\mu, i}(x)=\frac{\left|x-x_{i}\right|^{-\mu}}{\sum_{k=1}^{N}\left|x-x_{k}\right|^{-\mu}}
$$

and $|\cdot|$ denotes the Euclidean norm in $\mathbb{R}^{s}$. The interpolation operator $S_{N, \mu}[f]$ is stable, in the sense that

$$
\min _{i} f\left(x_{i}\right) \leq S_{N, \mu}[f](x) \leq \max _{i} f\left(x_{i}\right),
$$

but for $\mu>1$ the interpolating function $S_{N, \mu}[f](x)$ has flat spots in the neighborhood of all data points. Also, the degree of exactness of the operator $S_{N, \mu}[\cdot]$ is 0 , in the sense that if it is restricted to the polynomial space $P^{m}:=\{p: \operatorname{deg}(p) \leq m\}$, then $\left.S_{N, \mu}[\cdot]\right|_{P^{m}}=I d_{P^{m}}$ (the identity function on $P^{m}$ ) only for $m=0$. These drawbacks can be avoided by replacing each value $f\left(x_{i}\right)$ in (1.1) with an interpolation operator in $x_{i}$, applied to $f$, having a certain degree of exactness $m>0$. More precisely, if for each $i=1, \ldots, N P\left[\cdot, x_{i}\right]$ denotes such an interpolation operator in $x_{i}$, then the related combined Shepard operator is

$$
S_{N, \mu} P[f](x)=\sum_{i=1}^{N} A_{\mu, i}(x) P\left[f, x_{i}\right](x) .
$$

Received by the editor November 4, 2004 and, in revised form, June 3, 2005.

2000 Mathematics Subject Classification. Primary 41A05, 41A25; Secondary 65D05.

Key words and phrases. Univariate interpolation, combined Shepard operator, degree of exactness, rate of convergence. 
Shepard himself proposed such a generalization (in the bivariate case) by using for each $i=1, \ldots, N$ instead of $f\left(x_{i}\right)$ the Taylor polynomial of $f$ of degree 1 at $x_{i}$, when the gradient of $f$ at each node $x_{i}$ is also given. In this case the combined operator has degree of exactness 1 and if $\mu>1$ it also interpolates the first partial derivatives at the interpolation nodes 24.

Several interpolation operators have been introduced and studied to increase the degree of exactness of the Shepard operator: Taylor [7, 8, 14, 15, 24, Lagrange [8, 9, Hermite [5, 8, Birkhoff [6, 8, least square approximations 20, 21, 22, 25], and splines $[8$. The present paper is devoted to a further combination of the Shepard operator with a new interpolation operator, the generalized Taylor polynomial, recently proposed by F. Costabile [10. In section 2 we recall some known properties of the generalized Taylor polynomial and give new results on the error of approximation that will be used later in the paper. In section 3 we define the univariate Shepard-Bernoulli operator and study its rate of convergence when applied to functions in the class $C^{m}$ in an interval containing the nodes. This operator has degree of exactness equal to $m$, while the Shepard-Taylor, Shepard-Lagrange, and Shepard-Hermite operators all have degree of exactness equal to $m-1$. The considered operators all have the same rate of convergence; numerical examples, given in section 4, demonstrate the accuracy of the proposed combination in some special situations. Finally, in section 5 we apply the combined Shepard operators to the problem of interpolating the discrete solutions of initial value problems for ordinary differential equations. We interpolate the exact solution of such a problem by a rational (real analytic) function with an order of approximation that is comparable with that of the classical Hermite cubic spline interpolation. In our opinion this kind of approximation is desirable, in particular if the exact solution is analytic.

\section{Some Remarks about the generalized TAYlor POLYNomial}

The generalized Taylor polynomial is an expansion in Bernoulli polynomials, i.e., in the polynomials defined recursively by means of the following relations [18:

$$
\begin{cases}B_{0}(x)=1, & \\ B_{n}^{\prime}(x)=n B_{n-1}(x), & n \geq 1 \\ \int_{0}^{1} B_{n}(x) d x=0, & n \geq 1 .\end{cases}
$$

For functions in the class $C^{m}([a, b]), a, b \in \mathbb{R}, a<b$, this expansion is realized by the equation

$$
f(x)=P_{m}[f, a, b](x)+R_{m}[f, a, b](x), \quad x \in[a, b],
$$

where the polynomial approximant is defined by

$$
P_{m}[f, a, b](x)=f(a)+\sum_{k=1}^{m} \frac{B_{k}\left(\frac{x-a}{h}\right)-B_{k}}{k !} h^{k-1}\left(f^{(k-1)}(b)-f^{(k-1)}(a)\right)
$$

and the remainder term is

$$
R_{m}[f, a, b](x)=\frac{h^{m-1}}{m !} \int_{a}^{b} f^{(m)}(t)\left(B_{m}\left(\frac{b-t}{h}\right)-B_{m}\left(\frac{(x-t)-[x-t]}{h}\right)\right) d t
$$


where we have set $B_{k}=B_{k}(0)$ and we have denoted by [.] the integer part of the argument and $h=b-a$. The name chosen for the polynomial approximant $P_{m}[f, a, b]$ is derived from a nice property of this operator: its limit when $h \rightarrow 0$ is the Taylor polynomial $T_{m}[f, a](x)$ of degree $m$ of $f$ about $a$ :

$$
\lim _{h \rightarrow 0} P_{m}[f, a, b](x)=T_{m}[f, a](x) .
$$

Moreover, the polynomial $P_{m}[f, a, b]$ satisfies the following interpolation conditions:

$$
P_{m}[f, a, b](a)=f(a), \quad P_{m}[f, a, b](b)=f(b),
$$

and

$$
\frac{d^{k}}{d x^{k}} P_{m}[f, a, b](b)-\frac{d^{k}}{d x^{k}} P_{m}[f, a, b](a)=f^{(k)}(b)-f^{(k)}(a), \quad k=1, \ldots, m-1 .
$$

Another notable property of (2.3) is that, for each fixed $m \geq 1$, this approximant is exact for polynomials of degree not greater than $m$, since

$$
\int_{a}^{b}\left(B_{m}\left(\frac{b-t}{h}\right)-B_{m}\left(\frac{(x-t)-[x-t]}{h}\right)\right) d t=0
$$

for each $x \in[a, b]$.

Clearly a polynomial can be defined by means of equation (2.3) also in the case when $a>b$; a property of $P_{m}[f, a, b](x)$ not stated in [10] is that this polynomial does not depend on the order of the points $a, b$.

Theorem 2.1. For each $a, b \in \mathbb{R}, a \neq b$,

$$
P_{m}[f, b, a](x)=P_{m}[f, a, b](x), \quad \text { for each } m \geq 1, x \in \mathbb{R} .
$$

Proof. We will use the well-known symmetry of the Bernoulli polynomials [1]

$$
B_{n}(1-x)=(-1)^{n} B_{n}(x), n \geq 0, x \in \mathbb{R}
$$

and the property of the Bernoulli numbers [1]

$$
B_{n}=(-1)^{n} B_{n} \quad \text { for each } n \geq 2 \text {. }
$$


By a straightforward calculation we have

$$
\begin{aligned}
& P_{m}[f, b, a](x) \\
&= f(b)+\sum_{k=1}^{m} \frac{B_{k}\left(\frac{x-b}{a-b}\right)-B_{k}}{k !}(a-b)^{k-1}\left(f^{(k-1)}(a)-f^{(k-1)}(b)\right) \\
&= f(b)+\sum_{k=1}^{m} \frac{B_{k}\left(1-\frac{x-a}{b-a}\right)-B_{k}}{k !}(-1)^{k}(b-a)^{k-1}\left(f^{(k-1)}(b)-f^{(k-1)}(a)\right) \\
&= f(b)-\left(B_{1}\left(1-\frac{x-a}{b-a}\right)-B_{1}\right)(f(b)-f(a)) \\
&+\sum_{k=2}^{m} \frac{B_{k}\left(1-\frac{x-a}{b-a}\right)-B_{k}}{k !}(-1)^{k}(b-a)^{k-1}\left(f^{(k-1)}(b)-f^{(k-1)}(a)\right) \\
&= f(b)-\left(1-\frac{x-a}{b-a}\right)(f(b)-f(a)) \\
&+\sum_{k=2}^{m} \frac{(-1)^{k} B_{k}\left(\frac{x-a}{b-a}\right)-(-1)^{k} B_{k}}{k !}(-1)^{k}(b-a)^{k-1}\left(f^{(k-1)}(b)-f^{(k-1)}(a)\right) \\
&= P_{m}[f, a, b](x) .
\end{aligned}
$$

With regard to the successive derivatives of the polynomial $P_{m}[f, a, b](x)$ we can prove the following result.

Theorem 2.2. Let $f \in C^{m}([a, b])$ and suppose that the derivative $f^{(m+1)}$ exists and is bounded in $(a, b)$; set $h=b-a$. Then for each $j=1, \ldots, m$ we have

$$
\left.\frac{d^{j}}{d x^{j}} P_{m}[f, a, b](x)\right|_{x=a}=f^{(j)}(a)+O\left(h^{m-j+1}\right) .
$$

Proof. By differentiating $j$ times the polynomial $P_{m}[f, a, b](x)$ we have

$$
\frac{d^{j}}{d x^{j}} P_{m}[f, a, b](x)=\sum_{k=j}^{m} \frac{B_{k-j}\left(\frac{x-a}{h}\right)}{(k-j) !} h^{k-j-1}\left(f^{(k-1)}(b)-f^{(k-1)}(a)\right),
$$

so that

$$
\begin{aligned}
\left.\frac{d^{j}}{d x^{j}} P_{m}[f, a, b](x)\right|_{x=a} & =\sum_{k=j}^{m} \frac{B_{k-j}}{(k-j) !} h^{k-j-1}\left(f^{(k-1)}(b)-f^{(k-1)}(a)\right) \\
& =\sum_{k=0}^{m-j} \frac{B_{k}}{k !} h^{k-1}\left(f^{(k+j-1)}(b)-f^{(k+j-1)}(a)\right) .
\end{aligned}
$$

If $f \in C^{m}([a, b])$ and the derivative $f^{(m+1)}$ exists and is bounded in $(a, b)$, then by the well-known Taylor expansion with Lagrange remainder we have, for each $k=0,1, \ldots, m-j$,

$$
\begin{aligned}
h^{k-1}\left(f^{(k+j-1)}(b)\right. & \left.-f^{(k+j-1)}(a)\right) \\
& =\sum_{i=k+j}^{m} \frac{f^{(i)}(a)}{(i-k-j+1) !} h^{i-j}+\frac{f^{(m+1)}\left(\xi_{k}\right)}{(m-k-j+2) !} h^{m-j+1},
\end{aligned}
$$


for some $\xi_{k} \in(a, b)$. Consequently

$$
\begin{aligned}
& \left.\frac{d^{j}}{d x^{j}} P_{m}[f, a, b](x)\right|_{x=a} \\
& \quad=\sum_{k=0}^{m-j} \frac{B_{k}}{k !}\left(\sum_{i=k+j}^{m} \frac{f^{(i)}(a)}{(i-k-j+1) !} h^{i-j}+\frac{f^{(m+1)}\left(\xi_{k}\right)}{(m-k-j+2) !} h^{m-j+1}\right) \\
& =\sum_{k=0}^{m-j} \sum_{i=k+j}^{m} \frac{B_{k}}{k !} \frac{f^{(i)}(a)}{(i-k-j+1) !} h^{i-j}+\sum_{k=0}^{m-j} \frac{B_{k}}{k !} \frac{f^{(m+1)}\left(\xi_{k}\right)}{(m-k-j+2) !} h^{m-j+1} \\
& =\sum_{i=j}^{m}\left(\sum_{k=0}^{i-j} \frac{B_{k}}{k !(i-j+1-k) !}\right) f^{(i)}(a) h^{i-j}+\sum_{k=0}^{m-j} \frac{B_{k}}{k !} \frac{f^{(m+1)}\left(\xi_{k}\right)}{(m-k-j+2) !} h^{m-j+1} .
\end{aligned}
$$

Since [18, p. 233]

$$
\sum_{k=0}^{p} \frac{B_{k}}{k !(p+1-k) !}=\left\{\begin{array}{l}
1, \text { if } p=0 \\
0, \text { if } p \geq 1
\end{array}\right.
$$

we find

$$
\begin{aligned}
\left.\frac{d^{j}}{d x^{j}} P_{m}[f, a, b](x)\right|_{x=a} & =f^{(j)}(a)+\sum_{k=0}^{m-j} \frac{B_{k}}{k !} \frac{f^{(m+1)}\left(\xi_{k}\right)}{(m-k-j+2) !} h^{m-j+1} \\
& =f^{(j)}(a)+O\left(h^{m-j+1}\right) .
\end{aligned}
$$

Note that the polynomial $P_{m}[f, a, b]$ can be extended in a natural way to the whole real line. In order to obtain bounds for the remainder (2.4) even in points outside the interval $[a, b]$, we consider the operator

$$
f \rightarrow P_{m}[f, a, b]
$$

as acting on the space $C^{m}([c, d])$ with $c<a$ and $b<d$. Peano's kernel theorem [13. p. 70] provides an integral expression for the remainder (2.4), as indicated in the following theorem.

Theorem 2.3. Let $f \in C^{m}([c, d])$ and $x \in[c, d]$. Then for the remainder

$$
R_{m}[f, a, b](x)=f(x)-P_{m}[f, a, b](x)
$$

the following integral representations hold:

$$
R_{m}[f, a, b](x)= \begin{cases}\frac{1}{(m-1) !} \int_{x}^{b} f^{(m)}(t) K_{a, b}(x, t) d t, & c \leq x \leq a, \\ \frac{1}{(m-1) !} \int_{a}^{b} f^{(m)}(t) K_{a, b}(x, t) d t, & a \leq x \leq b, \\ \frac{1}{(m-1) !} \int_{a}^{x} f^{(m)}(t) K_{a, b}(x, t) d t, & b \leq x \leq d,\end{cases}
$$

where

$$
\begin{aligned}
K_{a, b}(x, t) & =(x-t)_{+}^{m-1}-(a-t)_{+}^{m-1} \\
& -\sum_{k=1}^{m} \frac{(m-1) !}{(m-k) !}\left[(b-t)_{+}^{m-k}-(a-t)_{+}^{m-k}\right] h^{k-1} \frac{B_{k}\left(\frac{x-a}{h}\right)-B_{k}}{k !}
\end{aligned}
$$

and $(\cdot)_{+}^{k}$ denotes the positive part of the kth power of the argument, i.e.,

$$
(s)_{+}^{k}=\max \left\{s^{k}, 0\right\} .
$$


Proof. First note that in the expansion (2.3) there are evaluations of derivatives of $f$ up to the order $m-1$ in points of $[c, d]$; on the other hand the exactness of the approximant (2.3) on the polynomial space $P^{m}$ implies the exactness of this operator on the space $P^{m-1}$. By applying Peano's kernel theorem, we then have

$$
R_{m}[f, a, b](x)=\frac{1}{(m-1) !} \int_{c}^{d} f^{(m)}(t) K_{a, b}(x, t) d t,
$$

where the kernel function (2.9) is obtained by applying the linear functional $f \rightarrow$ $R_{m}[f, a, b](x)$ to $(x-t)_{+}^{m-1}$ considered as a function of $x$. If $x \in[c, a]$, then by the additivity of the integral we have

$$
\begin{aligned}
R_{m}[f, a, b](x) & \\
= & \frac{1}{(m-1) !} \int_{c}^{x} f^{(m)}(t) K_{a, b}(x, t) d t+\frac{1}{(m-1) !} \int_{x}^{a} f^{(m)}(t) K_{a, b}(x, t) d t \\
& +\frac{1}{(m-1) !} \int_{a}^{b} f^{(m)}(t) K_{a, b}(x, t) d t+\frac{1}{(m-1) !} \int_{b}^{d} f^{(m)}(t) K_{a, b}(x, t) d t .
\end{aligned}
$$

Note that if $c<t<x$, then

$$
\begin{aligned}
K_{a, b}(x, t)= & (x-t)^{m-1}-(a-t)^{m-1} \\
& -\sum_{k=1}^{m} \frac{(m-1) !}{(m-k) !}\left[(b-t)^{m-k}-(a-t)^{m-k}\right] h^{k-1} \frac{B_{k}\left(\frac{x-a}{h}\right)-B_{k}}{k !}=0,
\end{aligned}
$$

because $(x-t)^{m-1}$ is a polynomial in $x$ of degree $m-1$ so that it must coincide with its generalized Taylor expansion (2.3). By definition of the positive part, the kernel (2.9) is also zero in the interval $b<t<d$, so we have the first case of (2.8).

The remaining expressions may be obtained by analogous arguments.

The previous theorem allows us to obtain the desired bounds.

Theorem 2.4. Let $f \in C^{m}([c, d])$ and $x \in[c, d]$. Then for the remainder (2.7) we have

$$
\left|R_{m}[f, a, b](x)\right| \leq \begin{cases}C(m)\left\|f^{(m)}\right\|(b-x)^{m}, & c<x<a, \\ C(m)\left\|f^{(m)}\right\|(b-a)^{m}, & a<x<b, \\ C(m)\left\|f^{(m)}\right\|(x-a)^{m}, & b<x<d,\end{cases}
$$

where $\|\cdot\|$ denotes the sup-norm on $[c, d]$ and

$$
C(m)=\frac{1}{m !}\left(1+\sum_{k=1}^{m} \sum_{l=1}^{k}\left(\begin{array}{c}
m \\
k
\end{array}\right)\left(\begin{array}{l}
k \\
l
\end{array}\right)\left|B_{k-l}\right|\right), \quad m=1,2, \ldots .
$$

Proof. Let us assume that $c<x<a$. In this case we have from (2.8) that

$$
\begin{aligned}
& R_{m}[f, a, b](x) \\
& \quad=\frac{1}{(m-1) !} \int_{x}^{a} f^{(m)}(t) K_{a, b}(x, t) d t+\frac{1}{(m-1) !} \int_{a}^{b} f^{(m)}(t) K_{a, b}(x, t) d t .
\end{aligned}
$$


If $x<t<a$, then

$$
\begin{aligned}
& K_{a, b}(x, t) \\
& \quad=-(a-t)^{m-1}-\sum_{k=1}^{m} \frac{(m-1) !}{(m-k) !}\left[(b-t)^{m-k}-(a-t)^{m-k}\right] h^{k-1} \frac{B_{k}\left(\frac{x-a}{h}\right)-B_{k}}{k !}
\end{aligned}
$$

so that

$$
\begin{aligned}
\int_{x}^{a} K_{a, b}(x, t) f^{(m)}(t) d t \\
=-\int_{x}^{a}(a-t)^{m-1} f^{(m)}(t) d t-\sum_{k=1}^{m} \frac{(m-1) !}{(m-k) !} h^{k-1} \frac{B_{k}\left(\frac{x-a}{h}\right)-B_{k}}{k !} \\
\quad \times \int_{x}^{a}\left((b-t)^{m-k}-(a-t)^{m-k}\right) f^{(m)}(t) d t
\end{aligned}
$$

Note that the integrands are of type $g(t) f^{(m)}(t)$ with a $g(t)$ that does not change sign in $[x, a]$. In view of this last property, by the first mean value theorem for integrals [13, Theorem 1.4.2] we have

$$
\begin{aligned}
\int_{x}^{a} K_{a, b}(x, t) f^{(m)}(t) d t \\
=-f^{(m)}(\xi) \int_{x}^{a}(a-t)^{m-1} d t-\sum_{k=1}^{m} \frac{(m-1) !}{(m-k) !} h^{k-1} \frac{B_{k}\left(\frac{x-a}{h}\right)-B_{k}}{k !} \\
\quad \times f^{(m)}\left(\xi_{k}\right) \int_{x}^{a}\left((b-t)^{m-k}-(a-t)^{m-k}\right) d t
\end{aligned}
$$

for some $\xi, \xi_{k} \in[c, b], k=1, \ldots, m$, so that we find after some calculations

$$
\begin{aligned}
\int_{x}^{a} K_{a, b}(x, t) f^{(m)}(t) d t \\
=-f^{(m)}(\xi) \frac{(a-x)^{m}}{m}-h^{m} \sum_{k=1}^{m} \frac{(m-1) !}{(m-k+1) !} \frac{B_{k}\left(\frac{x-a}{h}\right)-B_{k}}{k !} \\
\\
\times f^{(m)}\left(\xi_{k}\right)\left(-1+\sum_{j=0}^{m-k}\left(\frac{b-x}{h}\right)^{m-k-j}\left(\frac{a-x}{h}\right)^{j}\right) .
\end{aligned}
$$

If $a<t<b$, then

$$
K_{a, b}(x, t)=-\sum_{k=1}^{m} \frac{(m-1) !}{(m-i) !}(b-t)^{m-k} h^{k-1} \frac{B_{k}\left(\frac{x-a}{h}\right)-B_{k}}{k !}
$$

and

$$
\begin{aligned}
\int_{a}^{b} K_{a, b}(x, t) f^{(m)}(t) d t & \\
& =-\sum_{k=1}^{m} \frac{(m-1) !}{(m-i) !} h^{k-1} \frac{B_{k}\left(\frac{x-a}{h}\right)-B_{k}}{k !} \int_{a}^{b}(b-t)^{m-k} f^{(m)}(t) d t
\end{aligned}
$$


and by applying the first mean value theorem for integrals we find after some calculations that

$$
\int_{a}^{b} K_{a, b}(x, t) f^{(m)}(t) d t=-h^{m} \sum_{k=1}^{m} f^{(m)}\left(\eta_{k}\right) \frac{(m-1) !}{(m-k+1) !} \frac{B_{k}\left(\frac{x-a}{h}\right)-B_{k}}{k !}
$$

for some $\eta_{k} \in[c, b], k=1, \ldots, m$. Substituting into (2.11) the left-hand sides of (2.14), (2.15) with their respective right-hand sides, we finally obtain the following expression for the error:

$$
\begin{aligned}
R_{m}[f, a, b](x) & -f^{(m)}(\xi) \frac{(a-x)^{m}}{m !}-\frac{h^{m}}{m !} \sum_{k=1}^{m} f^{(m)}\left(\xi_{k}\right) \frac{m !}{(m-k+1) !} \frac{B_{k}\left(\frac{x-a}{h}\right)-B_{k}}{k !} \\
& \times\left(-1+\sum_{j=0}^{m-k}\left(\frac{b-x}{h}\right)^{m-k-j}\left(\frac{a-x}{h}\right)^{j}\right)-\frac{h^{m}}{m !} \sum_{k=1}^{m} f^{(m)}\left(\eta_{k}\right) \frac{m !}{(m-k+1) !} \\
& \times \frac{B_{k}\left(\frac{x-a}{h}\right)-B_{k}}{k !}
\end{aligned}
$$

In order to establish the bound (2.12) we make use of the well-known identities

$$
B_{k}(x)-B_{k}=\sum_{l=1}^{k}\left(\begin{array}{l}
k \\
l
\end{array}\right) B_{k-l} x^{l}, \quad k=1,2, \ldots,
$$

from which it results after some calculations

$$
\begin{aligned}
& \left|R_{m}[f, a, b](x)\right| \\
& \leq \frac{\left\|f^{(m)}\right\|}{m !}\left((a-x)^{m}+h^{m} \sum_{k=1}^{m} \sum_{l=1}^{k}\left(\begin{array}{c}
m \\
k
\end{array}\right)\left(\begin{array}{c}
k \\
l
\end{array}\right)\left|B_{k-l}\right|\left(\frac{b-x}{h}\right)^{m-(k-l)}\right) \\
& \quad \leq h^{m} \frac{\left\|f^{(m)}\right\|}{m !}\left(1+\sum_{k=1}^{m} \sum_{l=1}^{k}\left(\begin{array}{c}
m \\
k
\end{array}\right)\left(\begin{array}{c}
k \\
l
\end{array}\right)\left|B_{k-l}\right|\right)\left(\frac{b-x}{h}\right)^{m} .
\end{aligned}
$$

The third expression (2.12) can be proved in an analogous manner.

Since the degree of exactness of the operator $P_{m}[\cdot, a, b]$ is equal to $m$, we can prove the following result in an analogous manner.

Theorem 2.5. Let $f \in C^{m+1}([c, d])$ and $x \in[c, d]$. Then for the remainder (2.7) the inequalities

$$
\left|R_{m}[f, a, b](x)\right| \leq \begin{cases}C(m+1)\left\|f^{(m+1)}\right\|(b-x)^{m+1}, & c<x<a, \\ C(m+1)\left\|f^{(m+1)}\right\|(b-a)^{m+1}, & a<x<b, \\ C(m+1)\left\|f^{(m+1)}\right\|(x-a)^{m+1}, & b<x<d,\end{cases}
$$

hold with $C(m)$ defined in (2.13).

The first values of $C(m)$ for initial values of $m$ are given in Table 1 .

For large values of $m$ the constant $C(m)$ tends quickly to 0 ; in fact the following upper bound holds. 
TABLE 1. First values of $C(m)$

\begin{tabular}{|c|c|c|c|c|c|c|c|c|c|}
\hline$m$ & 1 & 2 & 3 & 4 & 5 & 6 & 7 & 8 & 9 \\
\hline$C(m)$ & 2.000 & 2.500 & 2.166 & 1.375 & 0.677 & 0.272 & 0.092 & 0.027 & 0.007 \\
\hline
\end{tabular}

Theorem 2.6. For each $m=1,2, \ldots$ the constant $C(m)$ defined in (2.13) satisfies the inequality

$$
\begin{aligned}
C(m) \leq \frac{1}{m !} & +2\left(\frac{m}{2}\right)^{1-\frac{m}{2}}+\left(\frac{m-1}{2}\right)^{1-\frac{m-1}{2}} \\
& +\frac{2 \pi^{2}}{3}\left[\frac{m-1}{2}\right] \frac{\left(\frac{m-2\left[\frac{m-31}{2}\right]}{2}\right)^{1-\frac{m-2\left[\frac{m-31}{2}\right]}{2}}}{(2 \pi)^{2\left[\frac{m-31}{2}\right]}} .
\end{aligned}
$$

Proof. Let us consider the sums involved in (2.13). By inverting their order and substituting $k-l$ with $s$ as a dummy index we have

$$
\begin{aligned}
\sum_{k=1}^{m} \sum_{l=1}^{k}\left(\begin{array}{c}
m \\
k
\end{array}\right)\left(\begin{array}{c}
k \\
l
\end{array}\right)\left|B_{k-l}\right| & =\sum_{l=1}^{m} \sum_{k=l}^{m}\left(\begin{array}{c}
m \\
k
\end{array}\right)\left(\begin{array}{c}
k \\
l
\end{array}\right)\left|B_{k-l}\right| \\
& =\sum_{l=1}^{m} \sum_{s=0}^{m-l}\left(\begin{array}{c}
m \\
l+s
\end{array}\right)\left(\begin{array}{c}
l+s \\
l
\end{array}\right)\left|B_{s}\right| \\
& =\sum_{s=0}^{m-1} \sum_{l=1}^{m-s}\left(\begin{array}{c}
m \\
l+s
\end{array}\right)\left(\begin{array}{c}
l+s \\
l
\end{array}\right)\left|B_{s}\right| .
\end{aligned}
$$

Since by [1]

$$
B_{0}=1, B_{1}=-\frac{1}{2}, B_{2 s+1}=0, s=1,2, \ldots,
$$

(2.13) becomes

$$
\begin{aligned}
C(m)=\frac{1}{m !}+\sum_{l=1}^{m} \frac{1}{m !}\left(\begin{array}{c}
m \\
l
\end{array}\right) & +\sum_{l=1}^{m-1} \frac{1}{2 m !}\left(\begin{array}{c}
m \\
l+1
\end{array}\right) l \\
& +\frac{1}{m !} \sum_{s=1}^{\left[\frac{m-1}{2}\right]_{l=1}^{m-2 s}}\left(\begin{array}{c}
m \\
l+2 s
\end{array}\right)\left(\begin{array}{c}
l+2 s \\
l
\end{array}\right)\left|B_{2 s}\right| .
\end{aligned}
$$

Now set

$$
C_{m, s}:=\frac{1}{m !} \sum_{l=1}^{m-2 s}\left(\begin{array}{c}
m \\
l+2 s
\end{array}\right)\left(\begin{array}{c}
l+2 s \\
l
\end{array}\right)\left|B_{2 s}\right| .
$$

By the formula [16]

$$
B_{2 s}=\frac{(-1)^{s-1} 2(2 s) !}{(2 \pi)^{2 s}} \zeta(2 s), s=1,2, \ldots,
$$

where, as usual, $\zeta$ denotes the Riemann zeta function, and by the inequality [16]

$$
n !>n^{\frac{n}{2}}, n=1,2, \ldots,
$$


we obtain after some calculations

$$
C_{m, s} \leq 2 \sum_{l=1}^{m-2 s} \frac{1}{(m-2 s-l)^{\frac{m-2 s-l}{2}} l^{\frac{l}{2}}} \times \frac{\zeta(2 s)}{(2 \pi)^{2 s}} .
$$

For each pair of fixed positive integers $m, s$, s.t. $m-2 s>0$, the function

$$
l \mapsto(m-2 s-l)^{\frac{m-2 s-l}{2}} l^{\frac{l}{2}}, \quad 0<l<m-2 s,
$$

attains its minimum value $\left(\frac{m-2 s}{2}\right)^{\frac{m-2 s}{2}}$ in $l=\frac{m-2 s}{2}$, so that we get

$$
C_{m, s} \leq 2 \sum_{l=1}^{m-2 s}\left(\frac{m-2 s}{2}\right)^{-\frac{m-2 s}{2}} \frac{\zeta(2 s)}{(2 \pi)^{2 s}},
$$

and since 16

$$
\zeta(2 s) \leq \zeta(2)=\frac{\pi^{2}}{6}, s=1,2, \ldots,
$$

we finally obtain

$$
C_{m, s} \leq 4\left(\frac{m-2 s}{2}\right)^{1-\frac{m-2 s}{2}} \frac{\frac{\pi^{2}}{6}}{(2 \pi)^{2 s}} .
$$

We can prove in an analogous manner that

$$
\sum_{l=1}^{m} \frac{1}{m !}\left(\begin{array}{c}
m \\
l
\end{array}\right) \leq 2\left(\frac{m}{2}\right)^{1-\frac{m}{2}}
$$

and

$$
\sum_{l=1}^{m-1} \frac{1}{2 m !}\left(\begin{array}{c}
m \\
l+1
\end{array}\right) l \leq\left(\frac{m-1}{2}\right)^{1-\frac{m-1}{2}} .
$$

A straightforward calculation furthermore shows that

$$
\begin{aligned}
\left(\frac{m-2 s}{2}\right)^{1-\frac{m-2 s}{2}} \frac{1}{(2 \pi)^{2 s}} & \leq \frac{\left(\frac{m-2\left[\frac{m-31}{2}\right]}{2}\right)^{1-\frac{m-2\left[\frac{m-31}{2}\right]}{2}}}{(2 \pi)^{2\left[\frac{m-31}{2}\right]}}, \\
m & =1,2, \ldots ; s=1,2, \ldots,\left[\frac{m-1}{2}\right],
\end{aligned}
$$

from which it easily follows that

$$
\sum_{s=1}^{\left[\frac{m-1}{2}\right]} 4\left(\frac{m-2 s}{2}\right)^{1-\frac{m-2 s}{2}} \frac{\frac{\pi^{2}}{6}}{(2 \pi)^{2 s}} \leq \frac{2 \pi^{2}}{3}\left[\frac{m-1}{2}\right] \frac{\left(\frac{m-2\left[\frac{m-31}{2}\right]}{2}\right)^{1-\frac{m-2\left[\frac{m-31}{2}\right]}{2}}}{(2 \pi)^{2\left[\frac{m-31}{2}\right]}}
$$

and then the assertion. 


\section{The Shepard-Bernoulli operator:}

DEFINITION, ERROR ESTIMATION, AND RATE OF CONVERGENCE

Let $x_{1}<x_{2}<\cdots<x_{N-1}<x_{N}$ be fixed points of an interval $I \subset \mathbb{R}$; we set $X=\left\{x_{1}, \ldots, x_{N}\right\}$ and $x_{N+1}=x_{N-1}$.

Definition 3.1. For each fixed $\mu>0$ and $m=1,2, \ldots$ the Shepard-Bernoulli univariate operator is defined by

$$
S_{B_{m}}[f](x)=\sum_{i=1}^{N} A_{\mu, i}(x) P_{m}\left[f, x_{i}, x_{i+1}\right](x), x \in I,
$$

where $P_{m}\left[f, x_{i}, x_{i+1}\right](x)$ is the natural extension of the polynomial approximant defined in (2.3) over the whole interval $I$.

The following statements can be checked without any difficulty.

Theorem 3.2. The operator $S_{B_{m}}[\cdot]$ is an interpolation operator in $x_{i}, i=1, \ldots, N$.

Proof. The assertion follows from the relations (2.5) in view of the well-known property

$$
A_{\mu, i}\left(x_{k}\right)=\delta_{i k}, \quad i, k=1, \ldots, N .
$$

Theorem 3.3. The degree of exactness of the operator $S_{B_{m}}[\cdot]$ is $m$, i.e., $S_{B_{m}}[p]=$ $p$ for each univariate polynomial $p \in P^{m}$.

Proof. The assertion follows from the well-known property

$$
\sum_{i=1}^{N} A_{\mu, i}(x) \equiv 1
$$

since the degree of exactness of $P_{m}\left[\cdot, x_{i}, x_{i+1}\right]$ is $m$ for $i=1, \ldots, N$.

As for the continuity class of the Shepard operator, and consequently the continuity class of the Shepard-Bernoulli operators, there is the following result [4].

Theorem 3.4. If $P\left[\cdot, x_{i}\right], i=1, \ldots, N$, are polynomial interpolation operators in $x_{i}$, then the continuity class of the operator (1.3) depends upon $\mu$ and, for $\mu>0$, is as follows:

i) if $\mu$ is an even integer, then $S_{N, \mu} P[\cdot] \in C^{\infty}$;

ii) if $\mu$ is an odd integer, then $S_{N, \mu} P[\cdot] \in C^{\mu-1}$;

iii) if $\mu$ is not an integer, then $S_{N, \mu} P[\cdot] \in C^{[\mu]}$;

here $[\mu]$ is the largest integer $<\mu$.

Remark 3.5. We emphasize that if $\mu$ is an even integer, then $S_{N, \mu} P[\cdot] \in C^{\omega}$ (the class of real analytic functions); in fact 8

$$
A_{\mu, i}(x)=\frac{\prod_{\substack{j=1 \\ j \neq i}}^{N}\left|x-x_{j}\right|^{\mu}}{\sum_{k=1}^{N} \prod_{\substack{j=1 \\ j \neq k}}^{N}\left|x-x_{j}\right|^{\mu}} .
$$

Theorem 3.6. For each $j$ s.t. $1 \leq j<\mu$ we have

$$
\left.\frac{d^{j}}{d x^{j}} S_{B_{m}}[f](x)\right|_{x=x_{k}}=\left.\frac{d^{j}}{d x^{j}} P_{m}\left[f, x_{k}, x_{k+1}\right](x)\right|_{x=x_{k}}, \quad k=1, . ., N .
$$


Proof. It is known that for each $j$ s.t. $1 \leq j<\mu$ ]

$$
\left.\frac{d^{j}}{d x^{j}} A_{\mu, i}(x)\right|_{x=x_{k}}=0, \quad i, k=1, \ldots, N .
$$

The assertion of the theorem then follows by applying the Leibniz rule and by using relations (3.2), (3.3):

$$
\begin{aligned}
& \left.\frac{d^{j}}{d x^{j}} S_{B_{m}}[f](x)\right|_{x=x_{k}} \\
& \quad=\left.\sum_{i=1}^{N} \frac{d^{j}}{d x^{j}}\left(A_{\mu, i}(x) P_{m}\left[f, x_{i}, x_{i+1}\right](x)\right)\right|_{x=x_{k}} \\
& \quad=\left.\left.\sum_{i=1}^{N} \sum_{l=0}^{j}\left(\begin{array}{l}
j \\
l
\end{array}\right) \frac{d^{j-l}}{d x^{j-l}} A_{\mu, i}(x)\right|_{x=x_{k}} \frac{d^{l}}{d x^{l}} P_{m}\left[f, x_{i}, x_{i+1}\right](x)\right|_{x=x_{k}} \\
& \quad=\left.\frac{d^{j}}{d x^{j}} P_{m}\left[f, x_{k}, x_{k+1}\right](x)\right|_{x=x_{k}} .
\end{aligned}
$$

In order to study the rate of convergence of the Shepard-Bernoulli operator we follow the ideas of Farwig's paper [15], later pursued in [8]. We introduce the following notations:

$$
\begin{gathered}
I_{\rho}(x)=[x-\rho, x+\rho], \rho>0, \\
r=\inf \left\{\rho>0: \forall x \in I, I_{\rho}(x) \cap X \neq \varnothing\right\},
\end{gathered}
$$

and

$$
M=\sup _{x \in I} \#\left(I_{r}(x) \cap X\right),
$$

where $\#(\cdot)$ denotes the cardinality function, so $M$ is the maximum number of points from $X$ contained in an interval $I_{r}(x)$. For the Shepard-Bernoulli univariate operator we then have the following error estimates.

Theorem 3.7. If $f \in C^{m}(I)$, then

$$
\left\|S_{B_{m}}[f]-f\right\| \leq C M\left\|f^{(m)}\right\| \varepsilon_{\mu}^{m-1}(r),
$$

where $\|\cdot\|$ denotes the sup norm in $I$,

$$
\varepsilon_{\mu}^{m-1}(r)= \begin{cases}|\log r|^{-1}, & \mu=1, \\ r^{\mu-1}, & 1<\mu<m+1, \\ r^{\mu-1}|\log r|, & \mu=m+1, \\ r^{m}, & \mu>m+1,\end{cases}
$$

$C$ is a positive constant independent of $x$ and $X$, and $r$ is given above.

Proof. Let $a, b \in I$ be fixed, $a<b$; for each $x \in I$ we set

$$
d[a, b](x)= \begin{cases}b-x, & x<a \\ b-a, & a \leq x \leq b, \\ x-a, & b<x\end{cases}
$$

i.e., $d[a, b](x)$ is the distance of $x$ from the interval $[a, b]$, plus $b-a$; we set also

$$
d^{m}[a, b](x)=(d[a, b](x))^{m} .
$$


In view of (2.12) the following inequality holds :

$$
\begin{aligned}
\left|S_{B_{m}}[f](x)-f(x)\right| & \leq\left|\sum_{i=1}^{N} A_{i}(x) P_{m}\left[f, x_{i}, x_{i+1}\right](x)-f(x)\right| \\
& \leq \sum_{i=1}^{N} A_{i}(x)\left|P_{m}\left[f, x_{i}, x_{i+1}\right](x)-f(x)\right| \\
& \leq C(m)\left\|f^{(m)}\right\|_{\infty} s_{\mu}^{m}(x),
\end{aligned}
$$

where

$$
s_{\mu}^{m}(x)=\frac{\sum_{i=1}^{N}\left|x-x_{i}\right|^{-\mu} d^{m}\left[x_{i}, x_{i+1}\right](x)}{\sum_{k=1}^{N}\left|x-x_{k}\right|^{-\mu}} .
$$

We want to show that

$$
s_{\mu}^{m}(x) \leq C M \varepsilon_{\mu}^{m-1}(r),
$$

where $C$ is a constant independent of $x$ and $X$. Denoting by diam $(I)$ the diameter of the interval $I$, we set

$$
\begin{gathered}
n=\left[\frac{\operatorname{diam}(I)}{2 r}\right]+1, \\
Q_{\rho}(u)=(u-\rho, u+\rho], \quad u \in I, \rho>0,
\end{gathered}
$$

and

$$
T_{j}=Q_{r}(x-2 r j) \cup Q_{r}(x+2 r j), \quad j=0,1, \ldots, n .
$$

Clearly the set $\bigcup_{j=-n}^{n} Q_{r}(x+2 r j)$ is a covering of $I$ with half open intervals; consequently, for each $i \in\{1, \ldots, N\}$ there exists a $j \in\{0, \ldots, n\}$ such that $x_{i} \in T_{j}$. Then the following inequalities hold:

$$
(2 j-1) r \leq\left|x-x_{i}\right| \leq(2 j+1) r
$$

and by (3.5)

$$
d\left[x_{i}, x_{i+1}\right](x) \leq(2(j+1)+1) r .
$$

On the other hand, by definition of $M$ it follows that

$$
1 \leq \#\left(X \cap T_{j}\right) \leq M
$$

for each $j=0,1, \ldots, n$. Let us denote by $x_{d}$ the node closest to $x$; since

$$
\frac{\left|x-x_{d}\right|^{-\mu}}{\sum_{k=1}^{N}\left|x-x_{k}\right|^{-\mu}} \leq 1
$$


we get, by applying (3.7), (3.8)

$$
\begin{aligned}
s_{\mu}^{m}(x) & \leq \sum_{x_{i} \in T_{0}} \frac{\left|x-x_{i}\right|^{-\mu} d^{m}\left[x_{i}, x_{i+1}\right](x)}{\sum_{k=1}^{N}\left|x-x_{k}\right|^{-\mu}}+\sum_{j=1}^{n} \sum_{x_{i} \in T_{j}} \frac{\left|x-x_{i}\right|^{-\mu} d^{m}\left[x_{i}, x_{i+1}\right](x)}{\sum_{k=1}^{N}\left|x-x_{k}\right|^{-\mu}} \\
& \leq \sum_{x_{i} \in T_{0}} d^{m}\left[x_{i}, x_{i+1}\right](x)+\left|x-x_{d}\right|^{\mu} \sum_{j=1}^{n} \sum_{x_{i} \in T_{j}}\left|x-x_{i}\right|^{-\mu} d^{m}\left[x_{i}, x_{i+1}\right](x) \\
& \leq M(3 r)^{m}+2 M r^{\mu} \sum_{j=1}^{n}((2 j-1) r)^{-\mu}((2 j+3) r)^{m} \\
& \leq M 5^{m} r^{m}\left(1+2 \sum_{j=1}^{n} j^{m-\mu}\right),
\end{aligned}
$$

where the last inequality follows from

$$
\begin{cases}2 j-1 \geq j, & j=1,2, \ldots \\ 2 j+3 \leq 5 j, & j=1,2, \ldots\end{cases}
$$

We have two cases:

Case $1(\mu>1)$.

Subcase 1a. If $1<\mu<m+1$, then

$$
r^{m}\left(1+2 \sum_{j=1}^{n} j^{m-\mu}\right)=O\left(r^{\mu-1}\right) .
$$

Subcase 1b. If $\mu=m+1$, then

$$
\sum_{j=1}^{n} j^{m-\mu}=\log n \geq|\log r| .
$$

Subcase 1c. If $\mu>m+1$, then $\sum_{j=1}^{n} j^{m-\mu}$ is bounded.

Case $2(\mu=1)$. The function $s_{1}^{m}(x)$ defined in (3.6),

$$
s_{1}^{m}(x)=\frac{\sum_{i=1}^{N}\left|x-x_{i}\right|^{-1} d^{m}\left[x_{i}, x_{i+1}\right](x)}{\sum_{k=1}^{N}\left|x-x_{k}\right|^{-1}}
$$

has its denominator greater than or equal to

$$
\sum_{x_{k} \in T_{0}}\left|x-x_{k}\right|^{-1}+\sum_{j=1}^{n} \sum_{x_{k} \in T_{j}}\left|x-x_{k}\right|^{-1} \geq \sum_{x_{k} \in T_{0}}\left|x-x_{k}\right|^{-1}+\frac{C}{r}|\log r|
$$

and its numerator less than or equal to

$$
\sum_{x_{i} \in T_{0}}\left|x-x_{i}\right|^{-1} d^{m}\left[x_{i}, x_{i+1}\right](x)+\sum_{j=1}^{n} \sum_{x_{i} \in T_{j}}\left|x-x_{i}\right|^{-1} d^{m}\left[x_{i}, x_{i+1}\right](x) .
$$

By applying the inequality

$$
\frac{\sum a_{i}}{\sum b_{i}} \leq \sum \frac{a_{i}}{b_{i}}, \quad a_{i} \geq 0, b_{i}>0
$$


we get

$$
\begin{aligned}
s_{1}^{m}(x) & \leq \frac{\sum_{x_{i} \in T_{0}}\left|x-x_{i}\right|^{-1} d^{m}\left[x_{i}, x_{i+1}\right](x)+\sum_{j=1 x_{i} \in T_{j}}^{n}\left|x-x_{i}\right|^{-1} d^{m}\left[x_{i}, x_{i+1}\right](x)}{\sum_{x_{k} \in T_{0}}\left|x-x_{k}\right|^{-1}+\frac{C}{r}|\log r|} \\
& \leq \sum_{x_{i} \in T_{0}} d^{m}\left[x_{i}, x_{i+1}\right](x)+\frac{C_{1} r}{|\log r|} \sum_{j=1}^{n} \sum_{x_{i} \in T_{j}}\left|x-x_{i}\right|^{-1} d^{m}\left[x_{i}, x_{i+1}\right](x) \\
& \leq M(3 r)^{m}+\frac{C_{1}}{|\log r|} \sum_{j=1}^{n} \sum_{x_{i} \in T_{j}}(2 j-1)^{-1}(2 j+3)^{m} r^{m} \\
& \leq M 5^{m} r^{m}\left(1+\frac{C_{2}}{|\log r|} \sum_{j=1}^{n} j^{m-1}\right) \\
& \leq M 5^{m} r^{m}\left(1+\frac{C_{2}}{|\log r|} O\left(r^{-m}\right)\right) \\
& =O\left(|\log r|^{-1}\right) .
\end{aligned}
$$

Remark 3.8. In the papers [15], 8] estimates similar to those of Theorem 3.7 have been proven for the Shepard-Taylor, Shepard-Lagrange, and Shepard-Hermite combinations, thus the rate of convergence is equal for all considered operators. Nevertheless we underline the following fact: for fixed $m \geq 1$ and $f \in C^{m}(I)$, the Shepard-Bernoulli operator has degree of exactness $m$, while the degree of exactness of Shepard-Taylor, Shepard-Lagrange, and Shepard-Hermite combinations is $m-1$.

In an analogous manner we can prove the following result:

Theorem 3.9. If $f \in C^{m+1}(I)$, then

$$
\left\|S_{B_{m}}[f]-f\right\|_{I} \leq C M\left\|f^{(m+1)}\right\| \varepsilon_{\mu}^{m}(r)
$$

where

$$
\varepsilon_{\mu}^{m}(r)= \begin{cases}|\log r|^{-1}, & \mu=1, \\ r^{\mu-1}, & 1<\mu<m+2, \\ r^{\mu-1}|\log r|, & \mu=m+2, \\ r^{m+1}, & \mu>m+2\end{cases}
$$

and $C$ is a positive constant independent of $x$ and $X$.

\section{Numerical tests}

Let us investigate the accuracy of the combined Shepard-Bernoulli approximation operator when applied to the following set of functions on the interval 


$$
I=[0,1]:
$$

$$
\begin{aligned}
\text { Saddle } f_{1}= & \frac{1.25}{6+6(3 x-1)^{2}}, \\
\text { Sphere } \quad f_{2}= & \frac{\sqrt{64-81(x-.5)^{2}}}{9}-.5 \\
\text { Cliff } \quad f_{3}= & \frac{\tanh (-9 x+1)}{2}+.5 \\
\text { Gentle } \quad f_{4}= & \frac{\exp \left(-\frac{81}{16}(x-.5)^{2}\right)}{3}, \\
\text { Steep } \quad f_{5}= & \frac{\exp \left(-\frac{81}{4}(x-.5)^{2}\right)}{3}, \\
\text { Exponential } \quad f_{6}= & .75 \exp \left(-\frac{(9 x-2)^{2}}{4}\right)+.75 \exp \left(-\frac{(9 x+1)^{2}}{49}\right) \\
& +.5 \exp \left(-\frac{(9 x-7)^{2}}{4}\right)+.2 \exp \left(-(9 x-4)^{2}\right) .
\end{aligned}
$$

These functions result from adapting to the univariate case test functions generally used in the multivariate interpolation of large sets of scattered data [19. For each function $f_{i}$ we will compare the numerical results obtained by applying the approximation $S_{B_{m}}[f]$ with those resulting from applying the following combined Shepard approximation operators known in literature: the Shepard-Lagrange univariate operator $S_{L_{m}}[f]$ and the Shepard-Taylor univariate operator $S_{T_{m}}[f]$ defined as follows $8,15,25]$ :

$$
S_{L_{m}}[f](x)=\sum_{i=1}^{N} A_{i}(x) L_{m}\left[f, x_{i}\right](x),
$$

with

$$
L_{m}\left[f, x_{i}\right](x)=\sum_{j=0}^{m} \prod_{\substack{k=0 \\ k \neq j}}^{m} \frac{x-x_{i+k}}{x_{i+j}-x_{i+k}} f\left(x_{i+j}\right), x_{N+j}=x_{N-m+j-1}, j=1, \ldots, m,
$$

and

$$
S_{T_{m}}[f](x)=\sum_{i=1}^{N} A_{i}(x) T_{m}\left[f, x_{i}\right](x)
$$

with

$$
T_{m}\left[f, x_{i}\right](x)=\sum_{j=0}^{m} \frac{f^{(j)}\left(x_{i}\right)}{j !}\left(x-x_{i}\right)^{j} .
$$

In the following section we will also consider a special case of the Shepard-Hermite univariate operator

$$
S_{H_{2 m-1}}[f](x)=\sum_{i=1}^{N} A_{i}(x) H_{2 m-1}\left[f, x_{i}\right](x),
$$


obtained from the Shepard operator in combination with the two-point Taylor interpolating polynomial [2, a special case of the Hermite interpolating polynomials:

$$
\begin{array}{r}
H_{2 m-1}\left[f, x_{i}\right](x) \\
=\sum_{j=0}^{m-1} \sum_{k=0}^{m-j-1}\left(\begin{array}{c}
m+k-1 \\
k
\end{array}\right)\left[\frac{\left(x-x_{i}\right)^{j}}{j !}\left(\frac{x-x_{i+1}}{x_{i}-x_{i+1}}\right)^{m}\left(\frac{x-x_{i}}{x_{i+1}-x_{i}}\right)^{k} f^{(j)}\left(x_{i}\right)\right. \\
\left.+\frac{\left(x-x_{i+1}\right)^{j}}{j !}\left(\frac{x-x_{i}}{x_{i+1}-x_{i}}\right)^{m}\left(\frac{x-x_{i+1}}{x_{i}-x_{i+1}}\right)^{k} f^{(j)}\left(x_{i+1}\right)\right] .
\end{array}
$$

Tables 2-7 show mean and maximum absolute interpolation errors, computed for different values of the parameters $\mu$ and $m$. Taking into account the number of degrees of freedom of each operator according to [3], we used uniform grids of 33 points in the interval $[0,1]$ for $S_{L_{0}}$ and $S_{T_{0}}$; at the same time we used grids of 17 points for $S_{B_{1}}, S_{L_{1}}$, and $S_{T_{1}}$, grids of 11 points for $S_{B_{2}}, S_{L_{2}}$, and $S_{T_{2}}$, and finally grids of 8 points for $S_{B_{3}}$. In order to have as accurate an estimation of the error as possible, we computed the resulting function approximations at the points

TABle 2. Saddle

\begin{tabular}{|c|r|l|r|l|r|l|}
\hline & \multicolumn{2}{|c|}{$S_{L_{m-1}}\left[f_{1}\right](x)$} & \multicolumn{2}{|c|}{$S_{T_{m-1}}\left[f_{1}\right](x)$} & \multicolumn{2}{|c|}{$S_{B_{m}}\left[f_{1}\right](x)$} \\
\hline$(\mu, m)$ & $\varepsilon_{\text {mean }}$ & $\varepsilon_{\text {max }}$ & $\varepsilon_{\text {mean }}$ & $\varepsilon_{\text {max }}$ & $\varepsilon_{\text {mean }}$ & $\varepsilon_{\text {max }}$ \\
\hline$(2,1)$ & 0.001328 & 0.003901 & 0.001328 & 0.003901 & 0.001050 & 0.004954 \\
\hline$(2,2)$ & 0.001050 & 0.004954 & 0.001067 & 0.003575 & 0.001062 & 0.004715 \\
\hline$(2,3)$ & 0.001248 & 0.007232 & 0.001043 & 0.003988 & 0.001490 & 0.005153 \\
\hline$(3,1)$ & 0.001246 & 0.002785 & 0.001246 & 0.002785 & 0.000476 & 0.003314 \\
\hline$(3,2)$ & 0.000476 & 0.003314 & 0.000321 & 0.002282 & 0.000333 & 0.002302 \\
\hline$(3,3)$ & 0.000718 & 0.004230 & 0.000151 & 0.001188 & 0.000206 & 0.001096 \\
\hline$(4,1)$ & 0.001437 & 0.003308 & 0.001437 & 0.003308 & 0.000457 & 0.003233 \\
\hline$(4,2)$ & 0.000457 & 0.003233 & 0.000244 & 0.001837 & 0.000259 & 0.001908 \\
\hline$(4,3)$ & 0.000699 & 0.004160 & 0.000052 & 0.000503 & 0.000136 & 0.001460 \\
\hline
\end{tabular}

TABle 3. Sphere

\begin{tabular}{|c|r|l|r|l|r|l|}
\hline & \multicolumn{2}{|c|}{$S_{L_{m-1}}\left[f_{2}\right](x)$} & \multicolumn{2}{|c|}{$S_{T_{m-1}}\left[f_{2}\right](x)$} & \multicolumn{2}{|c|}{$S_{B_{m}}\left[f_{2}\right](x)$} \\
\hline$(\mu, m)$ & $\varepsilon_{\text {mean }}$ & $\varepsilon_{\max }$ & $\varepsilon_{\text {mean }}$ & $\varepsilon_{\max }$ & $\varepsilon_{\text {mean }}$ & $\varepsilon_{\max }$ \\
\hline$(2,1)$ & 0.001330 & 0.006394 & 0.001330 & 0.006394 & 0.002145 & 0.005623 \\
\hline$(2,2)$ & 0.002145 & 0.005623 & 0.002263 & 0.005076 & 0.000312 & 0.000842 \\
\hline$(2,3)$ & 0.000260 & 0.000758 & 0.000371 & 0.000945 & 0.000586 & 0.002344 \\
\hline$(3,1)$ & 0.001408 & 0.004570 & 0.001408 & 0.004570 & 0.000583 & 0.001620 \\
\hline$(3,2)$ & 0.000583 & 0.001620 & 0.000480 & 0.001313 & 0.000058 & 0.000247 \\
\hline$(3,3)$ & 0.000106 & 0.000421 & 0.000034 & 0.000133 & 0.000079 & 0.000323 \\
\hline$(4,1)$ & 0.001628 & 0.005677 & 0.001628 & 0.005677 & 0.000510 & 0.001447 \\
\hline$(4,2)$ & 0.000510 & 0.001447 & 0.000287 & 0.000958 & 0.000039 & 0.000255 \\
\hline$(4,3)$ & 0.000096 & 0.000452 & 0.000007 & 0.000024 & 0.000025 & 0.000113 \\
\hline
\end{tabular}


TABle 4. Cliff

\begin{tabular}{|c|r|l|r|l|r|l|}
\hline & \multicolumn{2}{|c|}{$S_{L_{m-1}}\left[f_{3}\right](x)$} & \multicolumn{2}{|c|}{$S_{T_{m-1}}\left[f_{3}\right](x)$} & \multicolumn{2}{c|}{$S_{B_{m}}\left[f_{3}\right](x)$} \\
\hline$(\mu, m)$ & $\varepsilon_{\text {mean }}$ & $\varepsilon_{\max }$ & $\varepsilon_{\text {mean }}$ & $\varepsilon_{\max }$ & $\varepsilon_{\text {mean }}$ & $\varepsilon_{\text {max }}$ \\
\hline$(2,1)$ & 0.004773 & 0.039174 & 0.004773 & 0.039174 & 0.006604 & 0.038815 \\
\hline$(2,2)$ & 0.006604 & 0.038815 & 0.005827 & 0.019985 & 0.004710 & 0.031367 \\
\hline$(2,3)$ & 0.005537 & 0.046979 & 0.007020 & 0.025692 & 0.013455 & 0.062821 \\
\hline$(3,1)$ & 0.004080 & 0.026022 & 0.004080 & 0.026022 & 0.002522 & 0.021627 \\
\hline$(3,2)$ & 0.002522 & 0.021627 & 0.001727 & 0.015187 & 0.002466 & 0.027527 \\
\hline$(3,3)$ & 0.003962 & 0.035485 & 0.001144 & 0.011410 & 0.002138 & 0.016732 \\
\hline$(4,1)$ & 0.004661 & 0.030332 & 0.004661 & 0.030332 & 0.002405 & 0.021752 \\
\hline$(4,2)$ & 0.002405 & 0.021752 & 0.001347 & 0.013669 & 0.002170 & 0.034048 \\
\hline$(4,3)$ & 0.004146 & 0.040890 & 0.000663 & 0.008714 & 0.001542 & 0.024101 \\
\hline
\end{tabular}

TABle 5. Gentle

\begin{tabular}{|c|r|l|r|l|r|l|}
\hline & \multicolumn{2}{|c|}{$S_{L_{m-1}}\left[f_{4}\right](x)$} & \multicolumn{2}{|c|}{$S_{T_{m-1}}\left[f_{4}\right](x)$} & \multicolumn{2}{|c|}{$S_{B_{m}}\left[f_{4}\right](x)$} \\
\hline$(\mu, m)$ & $\varepsilon_{\text {mean }}$ & $\varepsilon_{\max }$ & $\varepsilon_{\text {mean }}$ & $\varepsilon_{\max }$ & $\varepsilon_{\text {mean }}$ & $\varepsilon_{\text {max }}$ \\
\hline$(2,1)$ & 0.002243 & 0.006798 & 0.002243 & 0.006798 & 0.002590 & 0.007116 \\
\hline$(2,2)$ & 0.002590 & 0.007116 & 0.002512 & 0.006030 & 0.001897 & 0.005956 \\
\hline$(2,3)$ & 0.001895 & 0.006919 & 0.001898 & 0.005585 & 0.001138 & 0.006015 \\
\hline$(3,1)$ & 0.002184 & 0.004667 & 0.002184 & 0.004667 & 0.000681 & 0.003277 \\
\hline$(3,2)$ & 0.000681 & 0.003277 & 0.000490 & 0.002431 & 0.000378 & 0.001727 \\
\hline$(3,3)$ & 0.000783 & 0.003035 & 0.000187 & 0.001007 & 0.000175 & 0.000940 \\
\hline$(4,1)$ & 0.002525 & 0.005541 & 0.002525 & 0.005541 & 0.000618 & 0.002978 \\
\hline$(4,2)$ & 0.000618 & 0.002978 & 0.000333 & 0.001689 & 0.000270 & 0.001163 \\
\hline$(4,3)$ & 0.000709 & 0.002656 & 0.000045 & 0.000249 & 0.000089 & 0.000575 \\
\hline
\end{tabular}

TABLE 6. Steep

\begin{tabular}{|c|r|l|r|l|r|l|}
\hline & \multicolumn{2}{|c|}{$S_{L_{m-1}}\left[f_{5}\right](x)$} & \multicolumn{2}{|c|}{$S_{T_{m-1}}\left[f_{5}\right](x)$} & \multicolumn{2}{|c|}{$S_{B_{m}}\left[f_{5}\right](x)$} \\
\hline$(\mu, m)$ & $\varepsilon_{\text {mean }}$ & $\varepsilon_{\max }$ & $\varepsilon_{\text {mean }}$ & $\varepsilon_{\max }$ & $\varepsilon_{\text {mean }}$ & $\varepsilon_{\text {max }}$ \\
\hline$(2,1)$ & 0.003744 & 0.012533 & 0.003744 & 0.012533 & 0.002358 & 0.012532 \\
\hline$(2,2)$ & 0.002358 & 0.012532 & 0.001790 & 0.007709 & 0.002950 & 0.015868 \\
\hline$(2,3)$ & 0.004414 & 0.027049 & 0.002697 & 0.011764 & 0.004950 & 0.019728 \\
\hline$(3,1)$ & 0.003060 & 0.008709 & 0.003060 & 0.008709 & 0.001930 & 0.011016 \\
\hline$(3,2)$ & 0.001930 & 0.011016 & 0.001218 & 0.006998 & 0.001501 & 0.009079 \\
\hline$(3,3)$ & 0.003487 & 0.017791 & 0.000586 & 0.003912 & 0.000909 & 0.005278 \\
\hline$(4,1)$ & 0.003527 & 0.010438 & 0.003527 & 0.010438 & 0.001945 & 0.011413 \\
\hline$(4,2)$ & 0.001945 & 0.011413 & 0.001000 & 0.005903 & 0.001323 & 0.008184 \\
\hline$(4,3)$ & 0.003478 & 0.018516 & 0.0002566 & 0.001789 & 0.000815 & 0.006381 \\
\hline
\end{tabular}

$i / 101, i=1, \ldots, 100$. Note that the approximation data corresponding to the classical Shepard operator coincide with those obtained by applying the operators 
TABLe 7. Exponential

\begin{tabular}{|c|r|l|l|l|r|l|}
\hline & \multicolumn{2}{|c|}{$S_{L_{m-1}}\left[f_{6}\right](x)$} & \multicolumn{2}{|c|}{$S_{T_{m-1}}\left[f_{6}\right](x)$} & \multicolumn{2}{|r|}{$S_{B_{m}}\left[f_{6}\right](x)$} \\
\hline$(\mu, m)$ & $\varepsilon_{\text {mean }}$ & $\varepsilon_{\max }$ & $\varepsilon_{\text {mean }}$ & $\varepsilon_{\max }$ & $\varepsilon_{\text {mean }}$ & $\varepsilon_{\text {max }}$ \\
\hline$(2,1)$ & 0.007930 & 0.025868 & 0.007930 & 0.025868 & 0.007669 & 0.034957 \\
\hline$(2,2)$ & 0.007669 & 0.034957 & 0.006299 & 0.022608 & 0.005271 & 0.025436 \\
\hline$(2,3)$ & 0.012072 & 0.045807 & 0.003968 & 0.019925 & 0.025296 & 0.067861 \\
\hline$(3,1)$ & 0.007543 & 0.018120 & 0.007543 & 0.018120 & 0.005122 & 0.021099 \\
\hline$(3,2)$ & 0.005122 & 0.021099 & 0.002905 & 0.015777 & 0.004379 & 0.024620 \\
\hline$(3,3)$ & 0.011187 & 0.048933 & 0.001660 & 0.010758 & 0.003523 & 0.020488 \\
\hline$(4,1)$ & 0.008484 & 0.020677 & 0.008484 & 0.020677 & 0.005026 & 0.022762 \\
\hline$(4,2)$ & 0.005026 & 0.022762 & 0.002402 & 0.013701 & 0.004233 & 0.024080 \\
\hline$(4,3)$ & 0.011221 & 0.051697 & 0.001198 & 0.008318 & 0.003020 & 0.018326 \\
\hline
\end{tabular}

$S_{L_{0}}$ or $S_{T_{0}}$; furthermore $S_{B_{1}}=S_{L_{1}}$. Numerical results show that the accuracy of the Shepard-Bernoulli interpolation is comparable with the accuracy of ShepardLagrange and Shepard-Taylor interpolation. To verify that the obtained results do not depend on the particular grid, we computed numerical approximations using sets of random nodes of interpolation in the interval $[0,1]$; the results show that the approximations are comparable in those cases, too.

\section{An application of the COMBined Shepard operators}

After solving initial value problems

$$
\begin{cases}y^{\prime}(x)=\varphi(x, y(x)), & (x, y) \in I \times \mathbb{R} \\ y\left(x_{0}\right)=y_{0}, & x_{0} \in I\end{cases}
$$

by means of a discrete method, we often need to know the solution on a set of points that differs from the grid. The nonnodal approximations are generally obtained by interpolation of the discrete data, and different features can be requested from the interpolants according to the problem. When the exact solution is infinitely many times differentiable (analytic), then the combined Shepard operators can be used to interpolate the discrete data. In fact, combinations of these operators with discrete solvers of ODEs will provide, for special values of $\mu$, infinitely differentiable (analytic) approximations of the solution of the problem (5.1) on the whole interval $I$. An algorithm for constructing these interpolants can be arranged as follows. The discrete solver produces an approximation $\tilde{y}_{i}$ of the exact solution $y\left(x_{i}\right)$, with a certain tolerance, at each node $x_{i}, i=1, \ldots, N$, in the interval $I$ of interest. Since a step control algorithm is enclosed in the ODE solver, the distribution of the nodes depends on the function $\varphi$. When $\varphi$ is smooth enough then $y \in C^{1}(I)$ (at least) and the value $\tilde{y}_{i}^{\prime}=\varphi\left(x_{i}, \tilde{y}_{i}\right)$ approximates the value $y^{\prime}\left(x_{i}\right), i=1, \ldots, N$, with the same precision as that of $\tilde{y}_{i}$ with respect to $y\left(x_{i}\right)$. The proposed interpolants are then obtained by substituting the exact values into the definition of the combined Shepard operators with their respective approximations.

Tables 8-11 show the accuracy of the combined Shepard operators $S_{T_{1}}[\cdot], S_{B_{2}}[\cdot]$, and $S_{H_{3}}[\cdot]$, when applied to interpolating the discrete solutions of the first four nonstiff DETEST problems A1-A4 [17]; a comparison of this accuracy to that of the classical cubic Hermite spline [3], here denoted by $C_{H_{3}}$, is possible. The 
discrete approximations are computed by means of the MATLAB ${ }^{\circledR}$ ode45 solver in the interval $[0,20]$ with various settings of the absolute and relative tolerances $\epsilon=\{$ AbsTol, RelTol $\}$. If we denote by $h_{\epsilon}=\max _{1 \leq n \leq N_{\epsilon}-1}\left|x_{n+1}^{\epsilon}-x_{n}^{\epsilon}\right|$ the size of the grid $0=x_{1}^{\epsilon}<x_{2}^{\epsilon}<\cdots<x_{N_{\epsilon}}^{\epsilon}=20$ of the nodal approximations $\tilde{y}=\left\{\tilde{y}_{i}\right\}$, then for $\mu=6, S_{T_{1}}[\tilde{y}](x), S_{B_{2}}[\tilde{y}](x)$, and $S_{H_{3}}[\tilde{y}](x)$ are rational, infinitely differentiable (analytic) functions, and, according to Theorem 3.9 and [8, Theorem 4],

$$
\begin{aligned}
& \left\|S_{T_{1}}[y]-y\right\| \leq C_{1}\left\|y^{(2)}\right\|_{I} h_{\epsilon}^{2}, \\
& \left\|S_{B_{2}}[y]-y\right\| \leq C_{2}\left\|y^{(3)}\right\|_{I} h_{\epsilon}^{3}, \\
& \left\|S_{H_{3}}[y]-y\right\| \leq C_{3}\left\|y^{(4)}\right\|_{I} h_{\epsilon}^{4} .
\end{aligned}
$$

Similarly, higher order approximations can be obtained by using the Shepard operator in combination with the osculatory interpolation polynomial that involves more than two points (for example three) [2, 3] or the operators $S_{T_{m-1}}[\cdot], S_{B_{m}}[\cdot]$, and $S_{H_{2 m-1}}[\cdot], m \geq 3$; but, in the last three cases, symbolic partial differentiations of the function defining the IVP problem are required. On the other hand it is well known [3] that $C_{H_{3}}$ is differentiable only once and

$$
\left\|C_{H_{3}}[y]-y\right\| \leq C_{4}\left\|y^{(4)}\right\|_{I} h_{\epsilon}^{4} .
$$

Problem A1: $\dot{y}=-y, y(0)=1$. Solution: $y(x)=e^{-x}$.

With the tolerances $\epsilon_{1}=\{1.0 e-06,1.0 e-03\}$ the MATLAB ${ }^{\circledR}$ ode 45 solver returns the discrete solution $\tilde{y}$ on a grid of 77 points and size $h_{\epsilon}=5.0 e-1$; by changing the error tolerances to $\epsilon_{2}=\{1.0 e-08,1.0 e-05\}$, ode 45 returns the discrete solution on a grid of 161 points and $h_{\epsilon}=4.2 e-1$; finally by setting $\epsilon_{3}=\{1.0 e-10,1.0 e-07\}$ ode 45 returns the discrete solution on a grid of 373 points and $h_{\epsilon}=2.4 e-1$; the maxima of the global errors computed on the output grid for $\tilde{y}$ and on the grid of 1001 points $i / 1001, i=1, \ldots, 1000$, with $S_{T_{1}}, S_{B_{2}}, S_{H_{3}}$, and $C_{H_{3}}$ are presented in the Table 8.

TABLE 8. Accuracy for the A1 DETEST problem

\begin{tabular}{|c|c|c|c|c|c|}
\hline & $\tilde{y}$ & $S_{T_{1}}$ & $S_{B_{2}}$ & $S_{H_{3}}$ & $C_{H_{3}}$ \\
\hline$\epsilon_{1}$ & $2.93 e-04$ & $4.43 e-03$ & $7.23 e-04$ & $2.96 e-04$ & $2.95 e-04$ \\
\hline$\epsilon_{2}$ & $2.49 e-06$ & $8.32 e-04$ & $2.56 e-05$ & $2.50 e-06$ & $2.50 e-06$ \\
\hline$\epsilon_{3}$ & $2.27 e-08$ & $1.20 e-04$ & $2.08 e-06$ & $2.69 e-08$ & $2.27 e-08$ \\
\hline
\end{tabular}

Problem A2: $\dot{y}=\frac{-y^{3}}{2}, y(0)=1$. Solution: $y(x)=\frac{1}{\sqrt{1+x}}$.

In this case and with the error tolerances $\epsilon_{1}=\{1.0 e-06,1.0 e-03\}$, the MATLAB ${ }^{\circledR}$ ode45 solver returns the discrete solution $\tilde{y}$ on a grid of 49 points and $h_{\epsilon}=5.0 e-1$; by changing the error tolerances to $\epsilon_{2}=\{1.0 e-08,1.0 e-05\}$, ode 45 returns the discrete solution on a grid of 65 points and $h_{\epsilon}=5.0 e-1$; finally by setting $\epsilon_{3}=\{1.0 e-10,1.0 e-07\}$, ode 45 returns the discrete solution on a grid of 117 points and $h_{\epsilon}=4.8 e-1$; the maxima of the global errors computed on the output grid for $\tilde{y}$ and on the grid of 1001 points for $S_{T_{1}}, S_{B_{2}}, S_{H_{3}}$, and $C_{H_{3}}$ are in the Table 9.

Problem A3: $\dot{y}=y \cos x, y(0)=1$. Solution: $y(x)=e^{\sin x}$.

In this case and with the error tolerances $\epsilon_{1}=\{1.0 e-06,1.0 e-03\}$, the MATLAB ${ }^{\circledR}$ ode45 solver returns the discrete solution $\tilde{y}$ on a grid of 93 points 
TABLE 9. Accuracy for the A2 DETEST problem

\begin{tabular}{|c|c|c|c|c|c|}
\hline & $\tilde{y}$ & $S_{T_{1}}$ & $S_{B_{2}}$ & $S_{H_{3}}$ & $C_{H_{3}}$ \\
\hline$\epsilon_{1}$ & $3.65 e-04$ & $1.61 e-03$ & $3.67 e-04$ & $3.70 e-04$ & $3.65 e-04$ \\
\hline$\epsilon_{2}$ & $2.85 e-06$ & $4.01 e-04$ & $2.85 e-05$ & $3.06 e-06$ & $2.85 e-06$ \\
\hline$\epsilon_{3}$ & $4.79 e-08$ & $7.29 e-05$ & $2.95 e-06$ & $9.06 e-08$ & $4.91 e-08$ \\
\hline
\end{tabular}

and $h_{\epsilon}=3.1 e-1$; by changing the error tolerances to $\epsilon_{2}=\{1.0 e-08,1.0 e-05\}$, ode 45 returns the discrete solution on a grid of 197 points and $h_{\epsilon}=1.5 e-1$; finally, by setting $\epsilon_{3}=\{1.0 e-10,1.0 e-07\}$, ode 45 returns the discrete solution on a grid of 465 points and $h_{\epsilon}=6.3 e-2$; the maxima of the global errors computed on the output grid for $\tilde{y}$ and on the grid of 1001 points for $S_{T_{1}}, S_{B_{2}}, S_{H_{3}}$, and $C_{H_{3}}$ are in the Table 10.

TABLE 10. Accuracy for the A3 DETEST problem

\begin{tabular}{|c|c|c|c|c|c|}
\hline & $\tilde{y}$ & $S_{T_{1}}$ & $S_{B_{2}}$ & $S_{H_{3}}$ & $C_{H_{3}}$ \\
\hline$\epsilon_{1}$ & $3.76 e-03$ & $2.54 e-02$ & $7.43 e-03$ & $3.79 e-03$ & $3.79 e-03$ \\
\hline$\epsilon_{2}$ & $7.49 e-05$ & $4.55 e-03$ & $6.73 e-04$ & $7.46 e-05$ & $7.46 e-05$ \\
\hline$\epsilon_{3}$ & $7.08 e-07$ & $7.65 e-04$ & $3.22 e-05$ & $8.72 e-07$ & $7.07 e-07$ \\
\hline
\end{tabular}

Problem A4: $\dot{y}=\frac{y}{4}\left(1-\frac{y}{20}\right), y(0)=1$. Solution: $y(x)=\frac{20}{1+19 e^{-x / 4}}$.

In this case and with the error tolerances $\epsilon_{1}=\{1.0 e-06,1.0 e-03\}$, the MATLAB ${ }^{\circledR}$ ode45 solver returns the discrete solution $\tilde{y}$ on a grid of 45 points and $h_{\epsilon}=5.0 e-1$; by changing the error tolerances to $\epsilon_{2}=\{1.0 e-08,1.0 e-05\}$, ode 45 returns the discrete solution on a grid of 49 points and $h_{\epsilon}=5.0 e-1$; finally, by setting $\epsilon_{3}=\{1.0 e-10,1.0 e-07\}$, ode 45 returns the discrete solution on a grid of 93 points and $h_{\epsilon}=3.6 e-1$; the maxima of the global errors computed on the output grid for $\tilde{y}$ and on the grid of 1001 points for $S_{T_{1}}, S_{B_{2}}, S_{H_{3}}$, and $C_{H_{3}}$ are in the Table 11.

TABLE 11. Accuracy for the A4 DETEST problem

\begin{tabular}{|c|c|c|c|c|c|}
\hline & $\tilde{y}$ & $S_{T_{1}}$ & $S_{B_{2}}$ & $S_{H_{3}}$ & $C_{H_{3}}$ \\
\hline$\epsilon_{1}$ & $4.64 e-05$ & $3.82 e-03$ & $3.57 e-04$ & $4.64 e-05$ & $4.64 e-05$ \\
\hline$\epsilon_{2}$ & $3.07 e-05$ & $3.81 e-03$ & $3.74 e-04$ & $3.07 e-05$ & $3.07 e-05$ \\
\hline$\epsilon_{3}$ & $2.05 e-06$ & $1.75 e-03$ & $1.26 e-04$ & $2.17 e-06$ & $2.05 e-06$ \\
\hline
\end{tabular}

Remark 5.1. The combined Shepard operators are global (i.e., their values at each point $x$ are affected by all the data); however, the global character of these interpolants can be avoided by substituting the weight functions $A_{\mu, i}(x)$ with basis functions $W_{\mu, i}(x)$ of an opportune class of differentiability $C_{0}^{s}(\mathbb{R}), 0 \leq s \leq \infty$, with small compact support and which may depend on the local distribution of data points [4, 23, 24, 26].

\section{Conclusions}

The univariate Shepard-Bernoulli operator can be extended to the multivariate case by using the expansions studied in [11, 12]. 


\section{ACKNOWLEDGMENTS}

The authors thank Professors F.A. Costabile and M. Redivo Zaglia for reading the paper and for useful suggestions. The authors are also grateful to the referees for their suggestions and remarks that were very useful in improving the paper.

\section{REFERENCES}

[1] M. Abramowitz, I. Stegun, Handbook of mathematical functions with formulas, graphs, and mathematical tables, Reprint of the 1972 edition, John Wiley \& Sons, Inc., New York, 1984. MR0757537 (85j:00005a)

[2] R.P. Agarwal, P.J.Y. Wong, Error inequalities in polynomial interpolation and their applications, Mathematics and its Applications, 262, Kluwer Academic Publishers, Dordrecht, 1993. MR1449390 (97m:41001)

[3] K.E. Atkinson, An Introduction to Numerical Analysis, John Wiley \& Sons, New York, 1978. MR0504339 (80a:65001)

[4] R.E. Barnhill, Representation and approximation of surfaces, in: Mathematical Software III, J.R. Rice, eds., Academic Press, New York (1977) 68-119. MR0489081 (58:8556)

[5] Gh. Coman, Hermite-type Shepard operators, Rev. Anal. Numér. Théor. Approx. 26 (1997) 33-38. MR1703917

[6] Gh. Coman, Shepard operators of Birkhoff-type, Calcolo 35 (1998) 197-203. MR1740751 (2001b:41026)

[7] Gh. Coman, L. Ţâmbulea, A Shepard-Taylor approximation formula, Studia Univ. BabeşBolyai Math. 33 (1998) 65-73. MR1027361 (90i:41003)

[8] Gh. Coman, R.T. Trîmbiţaş, Combined Shepard univariate operators, East J. Approx. 7 (2001) 471-483. MR1882130 (2002j:41018)

[9] Gh. Coman, R.T. Trîmbiţaş, Shepard operators of Lagrange-type, Studia Univ. Babeş-Bolyai Math. 42 (1997) 75-83.

[10] F. Costabile, Expansions of real functions in Bernoulli polynomials and applications, Conf. Semin. Mat. Univ. Bari 273 (1999) 1-13. MR1710747 (2000k:33014)

[11] F.A. Costabile, F. Dell'Accio, Expansion over a rectangle of real functions in Bernoulli polynomials and applications, BIT 41 (2001) 451-464. MR1854267|(2002g:65015)

[12] F. Costabile, F. Dell'Accio, Expansions over a simplex of real functions by means of Bernoulli polynomials, Numer. Algorithms 28 (2001) 63-86. MR1887748 (2003b:41024)

[13] P.J. Davis, Interpolation and Approximation, Dover Publications, Inc., New York, 1975. MR0380189 (52:1089)

[14] B. Della Vecchia, G. Mastroianni, On functions approximation by Shepard-type operatorsa survey, in: Approximation theory, wavelets and applications, S.P. Singh, eds., Kluwer Academic Publishers, Dordrecht (1995) 335-346. MR.1340899

[15] R. Farwig, Rate of convergence of Shepard's global interpolation formula, Math. Comp. 46 (1986) 577-590. MR0829627 (88a:65015)

[16] R.L. Graham, D.E. Knuth, O. Patashnik, Concrete Mathematics. A foundation for computer science, 2nd edition, Addison-Wesley Publishing Company, Reading, MA, 1994. MR1397498 (97d:68003)

[17] T.E. Hull, W.H. Enright, B.M. Fellen, A.E. Sedgwick, Comparing numerical methods for ordinary differential equations, SIAM J. Numer. Anal. 9 (1972) 603-637. MR0351086 (50:3577)

[18] R. Jordan, Calculus of Finite Differences, Chelsea Publishing Co., New York, 1960. MR0183987 (32:1463)

[19] R.J. Renka, A.K. Cline, A triangle-based $C^{1}$ interpolation method, Rocky Mountain J. Math. 14 (1984) 223-237. MR0736175 (85j:65005)

[20] R.J. Renka, Multivariate Interpolation of Large Sets of Scattered Data, ACM Trans. Math. Software 14 (1988) 139-148. MR0946761 (89d:65009)

[21] R.J. Renka, Algorithm 660, QSHEP2D: Quadratic Shepard Method for Bivariate Interpolation of Scattered Data, ACM Trans. Math. Software 14 (1988) 149-150.

[22] R.J. Renka, Algorithm 661, QSHEP3D: Quadratic Shepard Method for Trivariate Interpolation of Scattered Data, ACM Trans. Math. Software 14 (1988) 151-152. 
[23] L.L. Schumaker, Fitting surfaces to scattered data, in: Approximation Theory II, G.G. Lorentz, C.K. Chui, and L.L. Schumaker, eds., Academic Press, New York (1977) 203-268. MR0426369 (54:14312)

[24] D. Shepard, A two-dimensional interpolation function for irregularly-spaced data, in: Proceedings of the 1968 23rd ACM National Conference, ACM Press, New York (1968) 517-524.

[25] M.G. Trîmbiţaş, Combined Shepard-least square operators-computing them using spatial data structures, Studia Univ. Babeş-Bolyai Math. 47 (2002) 119-128. MR.1993911 (2004c:41045)

[26] C. Zuppa, Error estimates for modified local Shepard's interpolation formula, Appl. Numer. Math. 49 (2004) 245-259. MR2045501 (2005a:41020)

Dipartimento di Matematica, Università della Calabria, 87036 Rende (Cs), Italy

E-mail address: caira@unical.it

Dipartimento di Matematica, Università della Calabria, 87036 Rende (Cs), Italy

E-mail address: fdellacc@unical.it 